\title{
A Case of Subcutaneous and Gastrointestinal Basidiobolomycosis in Singapore
}

\author{
Teh Yii Ean, Tan Ban Hock, Limin Wijaya \\ Department of Infectious Diseases, Singapore General Hospital, Singapore
}

\begin{abstract}
Entomophthoromycosis due to Basidiobolus is a known cause of subcutaneous zygomycosis. It occasionally causes invasive disease involving the gastrointestinal tract. We describe a case of proven subcutaneous entomophthoromycosis, with subsequent development of gastrointestinal basidiobolomycosis, in a 67 year old Singaporean Chinese male. He presented with a progressively enlarging left inguinal mass with intra-abdominal extension, causing compression of the ureters and hydronephroses. He underwent multiple biopsies to ascertain the cause of the mass. Workup for malignancy, bacteria, fungal, parasitic and tuberculous infections were negative. He developed worsening gastrointestinal distension a year from initial presentation. Imaging showed mucosal thickening extending from the descending colon to the rectum. Based on the clinical presentation, he was treated as for basidiobolomycosis. He responded poorly to treatment and passed away two months after initiation of therapy. With the help of our counterparts in India, we proved our clinical suspicion by polymerase chain reaction on tissue specimen. J Microbiol Infect Dis 2017; 7(3):151-155
\end{abstract}

Keywords: Basidiobolomycosis, Entomophthoromycosis, Gastrointestinal, Inguinal mass

\section{INTRODUCTION}

Basidiobolus species are fungi that belong to the family Basidiobolaceae, of the order Entomophthorales. They are found in soil and decaying vegetable matter and as commensals in the gastrointestinal tract of amphibians, reptiles, fish and mammals [1]. Clinically, basidiobolomycosis causes chronic subcutaneous induration affecting the limbs and trunk. Rarely, it causes invasive disease involving the gastrointestinal tract or lungs. Minor trauma, local inoculation, and insect bites are the predominant modes of acquisition. Although widely prevalent in tropical and subtropical regions [2], it is infrequently seen in a developed country like Singapore. We discuss a case of a patient with proven subcutaneous entomopthoromycosis, who subsequently developed gastrointestinal symptoms, due to presumptive gastrointestinal basidiobolomycosis (GIB).

\section{CASE}

A 67 year old male engineer, with well-controlled diabetes mellitus, presented in July 2012 with left thigh swelling of three weeks duration. $\mathrm{He}$ did not recall any preceding bites or trauma. $\mathrm{He}$ had travelled frequently to rural areas of Thailand and Indonesia where he was involved in voluntary construction work. Initial imaging of the left thigh showed a $10 \times 6.3 \mathrm{~cm}$ lobulated soft tissue mass in the left inguinal region. Histology from biopsies of the mass showed granulomatous reaction and inflammatory cells including eosinophils. There was no eosinophilia. Blood films were negative for microfilaria. Smears from the biopsy were negative for fungi and acid fast bacilli (AFB) while bacteria, fungi and AFB cultures were eventually reported as negative. He defaulted subsequent clinic appointment.

He presented in November 2012 to another institute with persistent left thigh mass. He reported no new symptoms. However, a repeat scan showed left hydronephrosis. There was no 
intraabdominal extension of the left inguinal mass. He underwent cystoscopy, left double-J (DJ) stent insertion and bladder biopsy. Histology was unyielding with mild chronic inflammation with urothelial hyperplasia and focal cystitis cystica. There was no evidence of malignancy or lymphomatous infiltration. Histology from repeat left inguinal mass biopsy showed granulomatous inflammation with eosinophilic microabscesses. Quantiferon-TB Gold was negative. $\mathrm{He}$ was given empiric therapy with Ivermectin, Albendazole and Doxycycline for two weeks. He then sought alternative treatment. Filariasis $\mathrm{IgG}$ immunoassay performed at Westmead Hospital, Queensland returned to be negative a month later.

The patient was readmitted to our hospital in May 2013. By then, he had worsened with 20 kilograms of weight loss, worsening bilateral lower limb weakness and difficulty in passing urine for three months. He was found to have acute kidney injury with a creatinine of 1145 $\mu \mathrm{mol} / \mathrm{L}$. Further imaging showed marked progression of the left inguinal mass, with extension into the pelvis, causing distal ureteric obstruction and bilateral hydroureteronephroses. His kidney function improved after insertion of indwelling catheter and change of left DJ stent. Creatinine was $129 \mu \mathrm{mol} / \mathrm{L}$.

During the admission, a positron emission tomography (PET) and computerized tomography (CT) was performed. This showed hypermetabolic abnormal mucosal thickening extending from the descending colon to the rectum, in addition to the left inguinal mass (Figure 1). He underwent a colonoscopy where corresponding nodular mucosal thickening was seen. Histology revealed tubular adenoma with low grade dysplasia. No malignancy was identified.

He underwent an open incisional biopsy of the mass in June 2013. Histology showed granulomatous inflammation with patchy areas of eosinophilic infiltrate. In addition, structures with a clear central space containing finely granular material and rimmed by a purplish-red outer lining were seen within the stroma and engulfed within giant cells (Figure 2). There was no evidence of malignancy.
A presumptive diagnosis of entomopthoromycosis was made based on the clinical picture. He was started on Itraconazole on $12^{\text {th }}$ July and potassium iodide (KI) was added on 5th September. KI was stopped by $23^{\text {rd }}$ October in view of drug induced hypothyroidism and thyroxine replacement was started.

From $27^{\text {th }}$ July, his abdomen was noted to be increasingly distended. Abdominal CT showed nonspecific mural thickening of the colon and dilated colonic and small bowel loops with no transitional point to suggest extraluminal mechanical obstruction. The abdominal distension did not improve despite correction of electrolyte abnormalities, thyroxine replacement and antifungal agents. He was given intravenous neostigmine with good response and later oral pyridostigmine with minimal effect on motility. $\mathrm{He}$ was started on total parenteral nutrition in view of poor nutritional status from persistent ileus.

After a 2 month trial of antifungal therapy, a repeat abdominal CT showed interval increase in intestinal distension with no significant change in the mass. In view of limited response to treatment thus far and concern of poor absorption of Itraconazole due to persistent ileus, he was started on intravenous Voriconazole on $29^{\text {th }}$ October. This was oralised to Itraconazole on $4^{\text {th }}$ November due to worsening renal function. Terbinafine was added on $4^{\text {th }}$ November then stopped on 13th November in view of worsening renal impairment. Despite treatment, our patient did not show significant improvement. He collapsed suddenly and died on $21^{\text {st }}$ November.

Histological specimens of the left inguinal mass were sent to Postgraduate Institute of Medical Education and Research, Chandigarh, India for further evaluation which confers with the diagnosis of entomophthoromycosis. DNA identification was performed on tissue blocks obtained from open incisional biopsy. The fungus present was identified as Basidiobolus ranarum. Identification was by PCR with the Primer pair:

Forward:

Basidio(5'CTTTCGATCTGCCAGAGTT3')

Reverse:

ITS2(5'GCTGCGTTCTTCATCGATGC3') 
The BLAST result of the sequence obtained showed $98 \%$ similarity with the ITS region of Basidiobolus meristosporus (Basidiobolus ranarum) ATCC14450 in genbank database $(\mathrm{NCBI})$.
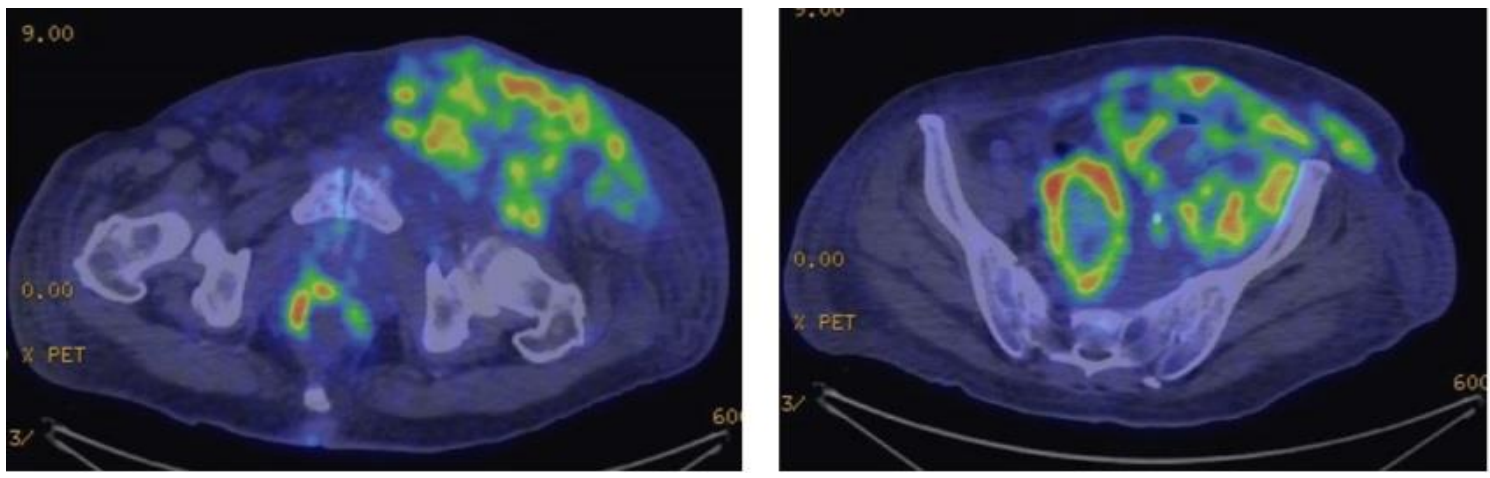

Figure1: PET CT showed hypermetabolic abnormal mucosal thickening extending from the descending colon to the rectum, in addition to the left inguinal mass.
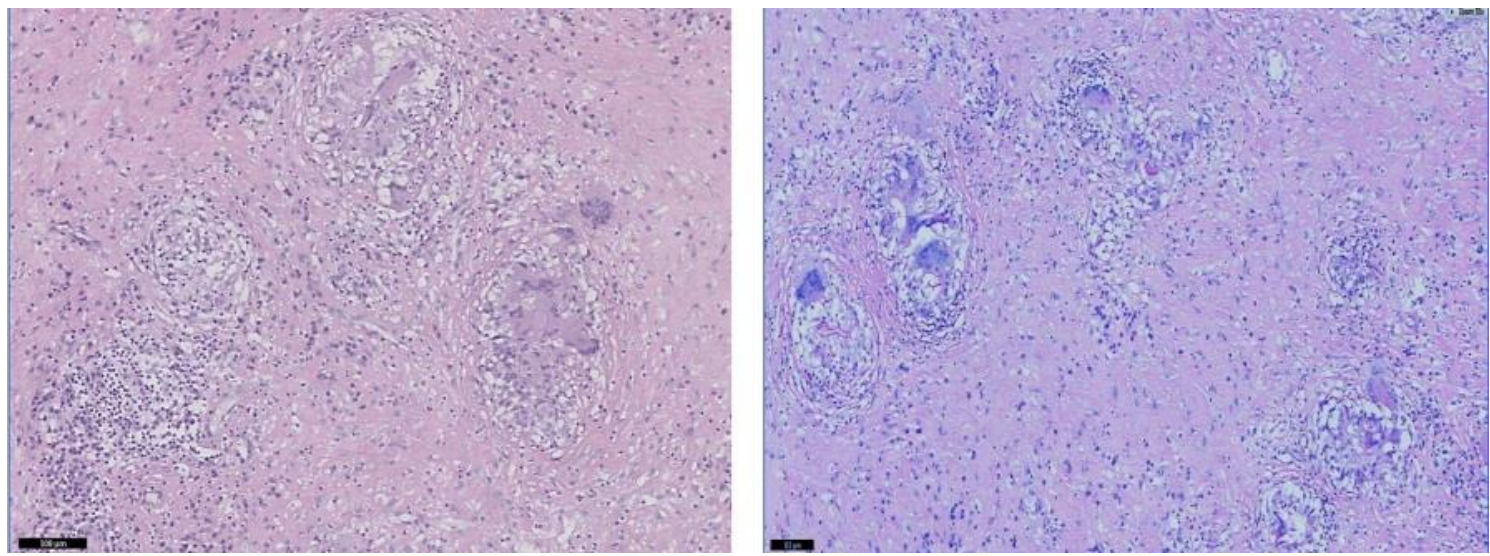

Figure2: Histology from incisional biopsy of the left groin mass showing granulomatous inflammation with patchy areas of eosinophilic infiltrate. Structures with a clear central space containing finely granular material and rimmed by a purplish-red outer lining are seen within the stroma and engulfed within giant cells.

\section{DISCUSSION}

To our knowledge, this is the first case of entomopthoromycosis reported in Singapore. This is also the first case report worldwide of a patient with proven subcutaneous entomopthoromycosis who subsequently developed presumptive GIB.

Entomopthoromycosis is a chronic nonangioinvasive fungal infection affecting immunocompetent individuals. Infections are caused by conidiobolus or basidiobolus species, with the latter being the most common cause of the disease[3]. There are no specific risk factors. In a case series from Arizona, 3 out of 7 had diabetes mellitus [4]. Our patient also had a history of diabetes mellitus, albeit it being wellcontrolled. Classically, infection results in skin and subcutaneous masses of the thighs and buttocks. Visceral involvement is unusual and so far only basidiobolus has been isolated from specimens from visceral organs [3].

GIB is not commonly reported in the literature and the total reported cases since 1964 till end 2014 were 52 cases worldwide[5-9]. Most cases were from areas with tropical and subtropical climates, mainly Africa and Asia. It is also an emerging infection in southwestern United States, with 19 cases reported through March 2012. 17 were residents of Arizona [5]. 
The most common presentation is abdominal pain. Other presenting symptoms are abdominal distension, constipation, abdominal mass, fever, weight loss, diarrhoea and vomiting. Classically, imaging show the presence of an intestinal mass, with the colon and the rectum being the most commonly affected. Non-specific bowel thickening, like that seen in our patient, is observed in about $25 \%$ of the patients on imaging studies[5]. Our patient had a PET/CT that showed hypermetabolic abnormal bowel thickening extending from the distal descending colon to the rectum and corresponding nodular mucosal thickening seen on colonoscopy. He had increasing abdominal distension, constipation and weight loss. These were suggestive of GIB. While histology did not show characteristic changes of entomopthoromycosis, this may be due to selective sampling during colonoscopy. He also had possible dissemination to the urinary tract, similar to the patient described by Khan et al [10]. However, we did not send urine samples for fungal isolation and hence this is presumptive.

Diagnosis of GIB is difficult due to the nonspecific clinical manifestations. It mimics common conditions like malignancy, inflammatory bowel disease and intestinal tuberculosis, resulting in delayed diagnosis and significant morbidity. A high index of suspicion is crucial, more so in developed countries where the condition is exceedingly rare.

Culture from the affected tissue is the gold standard for diagnosis. Macroscopically, colonies are yellow gray with a waxy appearance and many radial folds. Microscopically, there is granulomatous inflammation with marked tissue eosinophilia and Splendore-Hoeppli phenomenon surrounding broad branching, pleomorphic and sparsely septated hyphae [2]. In culture negative cases, serology and DNA sequencing from tissues are alternative modalities.

The current experience of treating patients with gastrointestinal basidiobolomycosis is limited to only a few cases. Surgery, in combination with a prolonged course of Itraconazole, seems to be the optimal treatment $[4,5,10]$. Surgical excision was not performed for our patient as the mass was too large; Surgery would be too extensive, requiring pelvectomy, removal of bladder, bowel and rectum. Moreover, it was soft and friable and technically difficult to excise completely. Ketoconazole [11], voriconazole [12] and posaconazole [13] have also been reported to be effective in a few cases. Amphotericin B was found to be active against only $50 \%$ of the isolates tested[1] and is less commonly used. Although $\mathrm{KI}$ has no known antifungal activity, it has been reported to be effective in treatment of subcutaneous basidiobolomycosis [14] and a case of retroperitoneal disease [15].

Prognosis is usually favorable with subcutaneous disease alone. In contrast, GIB is an aggressive disease. Vikram et al reported a $20 \%$ mortality rate in the largest review to date of 44 patients with GIB, some of whom underwent surgical resection and received antifungal therapy [7]. Our patient had a fatal outcome. This may be due to advanced disease and that surgical resection of the mass was not feasible given its extent. He also had advanced illness with involvement of the gastrointestinal tract. In addition, the time from first presentation to initiation of therapy was a year.

In conclusion, basdiobolomycosis is an emerging disease, especially with increased international travel. Diagnosis is often delayed due to its nonspecific presentation and myriad of differentials. This case report highlights the importance of increased awareness, prompt diagnosis and treatment of this disease, which will then translate to less morbidity and mortality.

\section{ACKNOWLEDGMENTS}

\section{Declaration of conflicting interests}

The authors declare that they have no conflict of interest.

\section{Financial disclosure}

No financial support was received.

\section{REFERENCES}

1. El-Shabrawi MHF, Kamal NM, Al-Harbi RJA, Voigt K, Al-Malki T. Gastrointestinal basidiobolomycosis: an emerging fungal infection causing bowel perforation in a child. $J$ Med Microbiol 2011;60:1395-1402.

Chapter in a book: Motoyama EK. Respiratory physiology in infants and children. In: Motoyama EK, Davis PJ, eds. Smith's Anesthesia for Infants and Children, 5th edn. St. Louis: C.V. Mosby, 1990:11-76. 
2. Chapter in a book: Kontoyiannis DP. Agents of Mucormycosis and Entomophthoramycosis. In: Mandell GL, Bennett JE, Dolin R, eds. Mandell, Douglas and Bennett's Principles and Practice of Infectious Diseases Volume 2, 7th edn. Philadelphia: Churchill Livingstone, 2010: 32663267.

3. Arjmand R, Karimi A, Dashti AS, Kadivar M. A Child with Intestinal Basidiobolomycosis. Iran J Med Sci 2012; 37(2):134-136.

4. Lyon GM, Smilack JD, Komatsu KK, et al. Gastrointestinal Basidiobolomycosis in Arizona: Clinical and Epidemiological Characteristics and Review of the Literature. Clin Infect Dis 2011; 32:1448-1455

5. Vikram HR, Smilack JD, Leighton JA, Crowell MD, De Petris G. Emergence of Gastrointestinal Basidiobolomycosis in the United States, With a Review of Worldwide Cases. Clin Infect Dis 2012;54(12):1685-1691.

6. Ejtehadi F, Anushiraviani A, Bananzadeh A, Geramizadeh B. Gastrointestinal Basidiobolomycosis Accompanied by Liver Involvement: A Case Report. Iran Red Cresent Med J 2014; 16(9):e14109.

7. Alsaleem K, Al-Mehaidib A, Banemai M, Hussain IB, Faqih M, Mehamadi A. Gastrointesinal basidiobolomycosis: mimicking Crohn's disease case report and review of the literature. Ann Saudi Med 2013; 33(5):500-504.

8. Alsheri $\mathrm{AH}$, Alsheri $\mathrm{A}$, Bawahab $\mathrm{MA}$, et al. Basidiobolomycosis: An Emerging Fungal Infection of the Gastrointestinal Tract in Adults. Am J Infect Dis 2013; 9(1):1-6.

9. Al-Maani AS, Paul G, Jardani A, et al. Gastrointestinal Basidiobolomycosis: First case report from Oman and literature review. Sultan Qaboos Univ Med J 2013; 14(2):241-244

10. Khan ZU, Khoursheed M, Makar R, et al. Basidiobolous ranarum as an Etiologic Agent of Gastrointestinal Zygomycosis. J Clin Microbiol 2001; 39(6):2360-2363

11. Janagond $A B$, Gouripur K, Dillirani V, Thnmozhivalli PR. Subcutaneous Basidiobolomcosis, an unusual case. Int $\mathrm{J}$ Biol Med Res 2013;4(1):2983-2985

12. Albaradi BA, Babiker AM, Al-Qahtani HS. Successful treatment of gastrointestinal basidiobolomycosis with voriconazole without surgical intervention. J Trop Pediatr 2014; 60(6):476-479

13. Rose SR, Lindsley MD, Hurst SF, Paddock CD, Damodaran T, Bennett J. Gastrointestinal basidiobolomycosis treated with posaconazole. Med Mycol Case Rep 2013; 2:11-14
14. Krishnan SG, Sentamilselvi G, Kamalam A, Das $\mathrm{KA}$, Janaki C. Entomophthoromycosis in India-a 4 year study. Mycoses 1998; 41(1-2):55-58.

15. Nazir Z, Hasan R, Pervaiz S, Alam M, Moazam F. Invasive retroperitoneal infection due to Basidiobolus ranarum with response to potassium iodide: case report and review of the literature. Ann Trop Paediatr 1997; 17:161-164. 